\title{
Modeling Asymmetric Volatility Clusters Using Copulas and High Frequency Data
}

\author{
Cathy Ning*a ${ }^{* a}$ Dinghai $\mathrm{Xu}^{b}$, and Tony S. Wirjanto ${ }^{c}$ \\ ${ }^{a}$ Department of Economics, Ryerson University,Toronto, Ontario, Canada, M5B 2K3. \\ ${ }^{b}$ Department of Economics, University of Waterloo, Waterloo, Ontario, Canada, N2L 3G1. \\ ${ }^{c}$ School of Accounting \& Finance and Department of Statistics \& Actuarial Science, \\ University of Waterloo, Waterloo, Ontario, Canada, N2L 3G1. \\ This Version: September 2009
}

*Corresponding author: Tel.: 1-416-979-5000 ext. 6181; Fax: 1-416-598-5916; E-mail address: qning@ryerson.ca. 


\begin{abstract}
Volatility clustering is a well-known stylized feature of financial asset returns. In this paper, we investigate the asymmetric pattern of volatility clustering on both the stock and foreign exchange rate markets. To this end, we employ copula-based semi-parametric univariate time-series models that accommodate the clusters of both large and small volatilities in the analysis. Using daily realized volatilities of the individual company stocks, stock indices and foreign exchange rates constructed from high frequency data, we find that volatility clustering is strongly asymmetric in the sense that clusters of large volatilities tend to be much stronger than those of small volatilities. In addition, the asymmetric pattern of volatility clusters continues to be visible even when the clusters are allowed to be changing over time, and the volatility clusters themselves remain persistent even after forty days.
\end{abstract}

Keywords: Volatility clustering, Copulas, Realized volatility, High-frequency data.

JEL Classification: C51, G32. 


\section{Introduction}

One of the best known stylized features of financial asset returns is volatility clustering. That is, a high (or low) volatility movement is observed to be followed by a high (or low) volatility movement. This volatility clustering captures the dynamics of volatility variation of financial assets. Modeling this volatility clustering of a financial asset is important since volatility values can directly impact prices of options and risks of stocks and portfolios. So far and to the best of our knowledge, volatility clustering has mainly been analyzed within the Generalized Autoregressive Conditional Heteroscedastic (GARCH) and Stochastic Volatility (SV) models. Although each class of these models can well capture the symmetric volatility clustering pattern of asset returns, both are somewhat limited in its ability to model the possible asymmetric pattern of the volatility clustering. This asymmetry arises from a frequent clustering of large volatilities of financial asset returns.

This paper contributes to the literature by investigating the possible asymmetry of volatility clustering of financial asset returns using a copula approach. In addition to modeling the symmetric volatility clustering, copulas also provide us with a flexible way to capture the possible asymmetry of the clusters of the volatilities. It is well known that copula is a multivariate distribution function of the standard uniform marginals. Due to Sklar's theorem, the dependence between variables can be modeled separately via certain copula functions and their corresponding marginals. This provides certain flexibility in the modeling exercise. In particular, different copulas are designed to capture different types of dependence structures: symmetric or asymmetric, and linear or nonlinear. Copula approach can also accommodate 
various types of marginal distributions. Due to its flexibility, the copula approach has been extensively applied in Finance in recent years. Just to mention a few, Chollete et al. (2006) and $\mathrm{Hu}$ (2006) use copula approaches and find asymmetric extreme dependence among equity returns, which indicates that the stock markets tend to crash together but do not boom together. Patton (2006) employs copulas to model the asymmetric dependence on the foreign exchange rate markets. He finds that both the Mark and Yen are more correlated when they are depreciating against the U.S. dollar than when they are appreciating. Jondeau and Rockinger (2006) use both the conditional and dynamic copula models to examine the dynamic dependence of the US and European stock market returns. Rodriguez (2006) investigates market contagions via copulas. A recent survey on applications of copulas in finance and economics can be found in Patton (2008).

While most applications of the copula approach are in the multivariate context, Chen and Fan (2006) develop theoretical foundations for extending the copula approach to model dependence of a univariate variable across time. Similar to that in Chen and Fan (2006), we apply the copula approach for a single time series variable and a semi-parametric estimation method to study the pattern of volatility clustering. The advantage of this method is that we do not need to specify the marginal distribution of the volatility, instead, we estimate the volatility with an empirical distribution function that is distribution-free. We focus on the volatility clustering, i.e., the dependence between volatilities at time $t$ and $t-1$, using parametric copulas. The copula parameters are estimated by a two-step maximum likelihood method, also known as the Canonical Maximum Likelihood (CLM) method. 
We note that copulas possess a number of attractive properties for the purpose of studying volatility dependence. First, copulas is designed to capture both nonlinear and linear dependence in a time series. This is especially important in the investigation of the dependence of the volatility clusters, which may not be linear. Second, copulas can accommodate any types of continuous marginal distributions, including a family of skewed and fat-tailed distributions, which can be used to characterize our volatility data with significant positive skewness and large excess kurtosis. Third, copulas are invariant to a strictly increasing transformation, including a nonlinear transformation. This feature is especially convenient since very often our transformation of the data is increasing but not linear. For further properties of copulas, see Joe (1997), Nelson (1999), Embrechts et al. (2002), and Cherubini et al. (2004).

In this paper, the characteristics of volatility dynamic clustering are examined for the returns from both the stock and foreign exchange rate markets. We construct daily realized volatilities using high frequency data. We find that, in our sample data, volatility clustering is mostly asymmetric, in the sense that volatility clustering is stronger for large volatilities than for small volatilities. This asymmetric pattern also exists in the dynamics of volatility clusters. This result reveals that, in addition to the volatility clustering found previously in the GARCH and SV models, the structure of the volatility clustering exhibits asymmetry, with an increasing tendency for clusters for large volatilities to occur than for small volatilities. This, to the best of our knowledge, has not been documented in the literature so far. This new result implies that consecutively highly volatile periods are more frequently 
observed on both the stock and currency markets than consecutively tranquil periods.

Our finding of asymmetric volatility clusters is consistent with the asymmetric leverage effect and volatility feedback effect documented in recent studies. Notably, Bollerslev et al. (2006) find a highly significant, prolonged leverage effect (i.e., volatility increases more following negative returns than positive returns.) and an almost instantaneous volatility feedback effect (i.e., an increase in volatility results in negative returns.) for the intra-daily data. Thus, through the leverage effect, a negative return initially increases subsequent volatility, and this, through the volatility feedback effect, induces a negative return. This negative return then, in turn, through the leverage effect, increases the subsequent volatility. The prolonged and asymmetric of these two effects implies a prolonged clustering of large volatilities.

In addition, in this paper, we also investigate the duration of the volatility clustering in the return series. Our results indicate that the clusters in most of the volatilities of the return series examined in this paper tend to be highly persistent and do not appear to dissipate even after forty days. This finding supports the long memory dependence in volatility documented in the literature, see Engle and Bollerslev (1986), Bollerslev et al. (1992), Ray and Tsay (2000) and among others.

The rest of the paper is organized as follows. Section 2 sets up the copula models for the volatility clustering. Section 3 describes the sample data and the construction of the realized volatility measures from the high frequency data. Section 4 presents and discusses the empirical results and Section 5 concludes. 


\section{The Methodology}

Copula is a multivariate cumulative distribution function with the marginal distribution being uniform on the interval $[0,1]$. In this paper, we apply copulas in modeling the dependence between consecutive volatilities of the return series. According to Sklar's (1959) theorem, there is a one-to-one relationship between a joint distribution and a copula. Chen and Fan (2006) extend this property from the multivariate context to a univariate set-up. Let $Y_{t}$ and $Y_{t-1}$ be the consecutive volatility variables at time $t$ and $t-1$ respectively. Then the clustering property of consecutive volatilities is completely characterized by their joint distribution, $H\left(y_{t}, y_{t-1}\right)$. In particular, based on Sklar's theorem, there exists a copula C(.) such that

$$
H\left(y_{t}, y_{t-1}\right)=C\left(u_{1}, u_{2}, \theta_{c}\right)
$$

where $u_{1}=G\left(y_{t}\right)$ and $u_{2}=G\left(y_{t-1}\right)$ are the marginal cumulative distribution functions of $Y_{t}$ and $Y_{t-1}$, respectively and $\theta_{c}$ is the copula parameter vector. In other words, the copula function is a joint distribution function of the transformed random variables $u_{1}=G\left(y_{t}\right)$ and $u_{2}=G\left(y_{t-1}\right)$. One advantage of copula approach is that it can separate the dependence from marginals, with the dependence completely captured in the copula function. Since our focus is on the dependence between two consecutive volatilities, rather than their marginals, we specify the copula function parametrically (but not the marginal distribution of the volatility). As a consequence, this particular approach is free of any specification errors for the marginals. This advantageous feature is highlighted in the simulation studies in Fermanian and Scaillet (2005). In this paper, we follow the framework in Chen and Fan (2006), which extends the method in Genest et al. (1995) from the i.i.d. multivariate 
time series to dependent univariate time series case, and use a semi-parametric estimation method (CML). See also in Cherubini et al. (2004)) and Joe (1997). In essence, this is a two-step procedure. In the first step, the marginal distribution function $G($.$) is estimated$ non-parametrically via its re-scaled empirical cumulative distribution function (ECDF)

$$
\widehat{G}\left(y_{t}\right)=\frac{1}{T+1} \sum_{t=1}^{T} 1\left\{Y_{t}<y\right\} .
$$

The ECDF is re-scaled to ensure that the first-order condition of the copula's log-likelihood function is well defined for all finite $T .{ }^{1}$ By the Glivenko-Cantelli theorem, $\widehat{G}_{Y}\left(y_{t}\right)$ converges to its theoretical counterpart $G\left(y_{t}\right)$ uniformly. In the second step, given the nonparametrically estimated ECDF, $\widehat{G}\left(y_{t}\right)$ and $\widehat{G}\left(y_{t-1}\right)$, we can estimate the copula parameters $\theta_{c}$ parametrically by the method of maximum likelihood, with

$$
\begin{aligned}
\widehat{\theta}_{c} & =\arg \max _{\theta_{c}} \widetilde{L} \\
\text { where } \widetilde{L}\left(\theta_{c}\right) & =\frac{1}{T} \sum \log c\left(\widehat{G}\left(y_{t}\right), \widehat{G}\left(y_{t-1}\right) ; \theta_{c}\right),
\end{aligned}
$$

where c(.) is the copula density function. Joe (1997) proves that under a set of regularity conditions, the two-step estimator is consistent and asymptotically normal. It is also pointed out that the two-step method is highly efficient. In addition, as indicated in Patton (2008), this method has the benefit of being computationally tractable. Importantly Chen and Fan (2006) establish the asymptotic properties for this semi-parametric estimator.

Using copulas, we can measure the dependence at the extremes by the extent of the tail dependence. A tail dependence measures the probability that both variables are in their

\footnotetext{
${ }^{1}$ See Genest et al. (1995) and Chen and Fan (2006) for a further discussion on this.
} 
lower or upper joint tails. Intuitively, an upper (lower) tail dependence refers to the relative amount of mass in the upper (lower) quantile of their joint distribution. The lower (left) and upper (right) tail dependence coefficients, $\lambda_{l}$ and $\lambda_{r}$, in the context of volatility dependence (clusters) are defined as:

$$
\begin{gathered}
\lambda_{l}=\lim _{u \longrightarrow 0} \operatorname{Pr}\left[G\left(y_{t}\right) \leq u \mid G\left(y_{t-1}\right) \leq u\right]=\lim _{u \longrightarrow 0} \frac{C(u, u)}{u}, \\
\lambda_{r}=\lim _{u \longrightarrow 1} \operatorname{Pr}\left[G\left(y_{t}\right) \geq u \mid G\left(y_{t-1}\right) \geq u\right]=\lim _{u \longrightarrow 1} \frac{1-2 u+C(u, u)}{1-u},
\end{gathered}
$$

respectively, where $\lambda_{l}$ and $\lambda_{r} \in[0,1]$. If $\lambda_{l}$ or $\lambda_{r}$ is positive, $Y_{t}$ and $Y_{t-1}$ are said to be left (lower) or right (upper) tail dependent. That is, $\lambda_{l}$ measures the degree of clusters of low volatilities while $\lambda_{r}$ measures the degree of clusters of high volatilities. Since tail dependence measures are derived from the copula functions, they possess all desirable properties of copulas mentioned earlier.

Different copulas represent different dependence structures with the so called association parameters $\theta_{c}$ which indicates the strength of the dependence. To capture the volatility dependence, we need to use a copula that allows for both the left tail (to capture clusters of small volatilities) and right tail dependence (to capture clusters of large volatilities). Thus we use the Symmetrized Joe Clayton (SJC) copula that was first introduced and used in Patton (2006). The SJC copula allows for asymmetric upper and lower tail dependence and symmetric dependence as a special case. It is defined as

$$
C_{S J C}\left(u, v \mid \lambda_{r}, \lambda_{l}\right)=0.5 \times\left(C_{J C}\left(u, v \mid \lambda_{r}, \lambda_{l}\right)+C_{J C}\left(1-u, 1-v \mid \lambda_{l}, \lambda_{r}\right)+u+v-1\right),
$$

where $C_{J C}\left(u, v \mid \lambda_{r}, \lambda_{l}\right)$ is the BB7 copula (also called Joe-Clayton copula) of Joe (1997) 
defined as

$$
\begin{aligned}
& C_{J C}\left(u, v \mid \lambda_{r}, \lambda_{l}\right) \\
= & 1-\left(1-\left\{\left[1-(1-u)^{k}\right]^{-r}+\left[1-(1-v)^{k}\right]^{-r}-1\right\}^{-1 / r}\right)^{1 / k},
\end{aligned}
$$

where $k=1 / \log _{2}\left(2-\lambda_{r}\right), \quad r=-1 / \log _{2}\left(\lambda_{l}\right)$, and $\lambda_{l} \in(0,1), \lambda_{r} \in(0,1)$. By construction, the SJC copula is symmetric when $\lambda_{l}=\lambda_{r}$.

We also examine the possibility of dynamic or time varying tail dependence in the data. In particular, following Patton (2006), we estimate the following ARMA-type process for tail dependence:

$$
\begin{aligned}
& \lambda_{l, t}=\left(1+\exp \left(-h_{l, t}\right)\right)^{-1}, \quad \lambda_{r, t}=\left(1+\exp \left(-h_{r, t}\right)\right)^{-1}, \\
& h_{l, t}=h_{l, 0}+\beta_{l} h_{l, t-1}+\alpha_{l} \sum_{j=1}^{p}\left|u_{t-j}-v_{t-j}\right|, \\
& h_{r, t}=h_{r, 0}+\beta_{r} h_{r, t-1}+\alpha_{r} \sum_{j=1}^{p}\left|u_{t-j}-v_{t-j}\right| .
\end{aligned}
$$

This model contains an autoregressive term designed to capture persistence in dependence, and a variable which is a mean absolute difference between $u$ and $v$. The latter variable is positive when the two probability integral transforms are on the opposite side of the extremes of the joint distribution and close to zero when they are on the same side of the extremes. The logistic transformation of the ARMA process guarantees that the tail dependence parameters lie in the $[0,1]$ interval. 


\section{Data Description and Construction of Realized Volatil-}

\section{ity Measure}

It is well-known that volatility can not be directly observed on the financial market. In this paper, a model-free measurement for volatility is constructed by using the intra-daily highfrequency data as a proxy for the volatility, which is known as realized volatility. Asymmetric volatility clusters are then examined on both the stock and foreign exchange rate markets. Our sample data set consists of five individual company stocks, including Yahoo (YHOO), Amazon (AMAZ), Microsoft (MSFT), Costco (COST) and IBM (IBM); two stock indices, including the S\&P 500 (SPX) and Dow Jones Industrial Average (INDU) and four foreign exchange rates, including Canadian dollar (CADA), Great British pound (GBPA), German mark (DEMA) and Japanese yen (JPYA). ${ }^{2}$

A common model-free indicator of the daily volatility is a simple summation of squared intra-day returns, see Andersen and Bollerslev (1998), Barndorff-Nielsen and Shephard (2001) and etc. To set forth the notation, let $p_{d, t}$ be the logarithmic price at a certain sampling frequency interval on day $t$. Consequently, the continuously compounded returns with $D$ observations on day $t$ is defined as,

$$
r_{d, t}=100\left(p_{d, t}-p_{d-1, t}\right)
$$

\footnotetext{
${ }^{2}$ All the currencies are in terms of the US dollars. The sample interval covers different periods depending on the data availability. The company stocks data are from June 06, 2005 to September 19, 2008. The foreign exchange rates data range from January 4, 1999 to December 30, 2005. The stock indices are from January 2, 1998 to December 30, 2005. Our data source is www.tickplusdata.com.
} 
where $d=1,2, \ldots, D$ and $t=1,2, \ldots, T$. When $D=1$, the first subscript is ignored and $r_{t}$ denotes the return series on a given day.

As mentioned earlier, a simple estimator of the daily volatility can be constructed by summing up the squared intra-day returns when the market is open, i.e.,

$$
R V_{t}=\sum_{d=1}^{D} r_{d, t}^{2}
$$

Under the assumptions that returns have zero mean, zero correlation and have finite second moments, (11) can be considered as a consistent measure of the daily volatility under an ideal market condition. However, in practice, there are two potential issues in the construction for the realized volatility. Due to a rapid development in the computer technology, the financial transaction data have become available at the ultra high frequency level. In particular, the transaction price nowadays can be recorded at a very fine time scale, such as transaction by transaction (tick-by-tick), one minute, five minute and etc. This raises the question of which set of discrete time series data we should choose as a measure of the realized volatility. The choice of the sampling frequency is not trivial. Intuitively, a larger data set should always contain more information. As the sampling interval approaches zero, the realized volatility can be equivalently considered as an integrated estimator. However, there is an important trade-off for choosing the observation frequency due to the presence of the market microstructure contaminations. In other words, financial asset prices diverge from their "efficient values" due to a variety of market frictions. Recently, increasing attention has been focused on the analysis of the realized volatility measures, see for instance Zhang et al. (2005), Hansen and Lunde (2006), van Dijk and Martens (2007), Andersen, Bollerslev 
and Diebold (2007), Maheu and McCurdy (2009) and etc. See also a recent survey paper on this topic by McAleer and Medeiros (2008). In this paper, we follow most of the literature and simply use 5-minute sampling intra-day data because the 5 -minute interval is considered as the frequency at which the transaction prices are less distorted from the microstructure noises.

Another non-trivial issue in the construction of (11) is the non-trading period effect. In the real market and to the extent that the markets are open only during a certain period of the trading days. (11) only measures the open-to-close volatility. This ignores information from the non-trading hours (e.g., overnight). To accommodate this overnight effect, Martens (2002), Koopman, Jungbacker and Hol (2005) and Hansen and Lunde (2006) propose a scaling (weighting) volatility estimator based on (11). The basic idea of this is to incorporate non-trading hours information into the construction of the variable. In this paper, we follow Martens (2002) and introduce an informative weighting factor in (11). In particular, we construct the measure of realized volatility as:

$$
R V_{t}=\delta \sum_{d=1}^{D} r_{d, t}^{2}
$$

where $\delta=1+\frac{w_{1}}{w_{2}}$, with $w_{1}=\frac{1}{T} \sum_{t=1}^{T} 100^{2}\left(p_{D, t}-p_{0, t}\right)^{2}$ and $w_{2}=\frac{1}{T} \sum_{t=1}^{T} 100^{2}\left(p_{0, t}-p_{D, t-1}\right)^{2}$. Then we use (12) to construct the measure of realized volatilities based on the 5-minute sampling frequency.

The summary statistics of the volatilities are presented in Table 1 . We note that the constructed realized volatilities are all right-skewed, indicating a longer right tail than the left one. The kurtosis ranges from 13.5 to 127.9, showing a significant fat-tail property 
of the return distribution. It is important to stress that the dependence of non-normally distributed variables can not be properly measured by a linear correlation method. The copula approach, on the other hand, which allows for any continuous marginal distributions, is expected to provide a more accurate measure for this type of dependence in the data.

\section{Empirical Results}

In this section, we discuss the results obtained from applying the approach described in section 2 to our realized volatility series. First, we consider the linear correlation measures of the consecutive volatilities in the first row of Table 2. The reported correlation coefficients range from 0.22 to 0.69 , reflecting a modest linear dependence between the consecutive volatilities of the return series. This represents a stylized feature of the volatility clustering of the returns on both the stock and foreign exchange rate markets.

Next, we examine the dependence structure between volatility $Y_{t}$ at $t$ and $Y_{t-1}$ at $t-1$ via the SJC copula, in which the tail dependence parameters are estimated directly. The results are presented in Table 2. First, both the left and right tail dependence parameters are observed to be highly, statistically significant, indicating the existence of both the left and right tail dependence of the consecutive volatilities; this means the probability of the clusters of small volatilities with small volatilities, and large volatilities with large volatilities. Second, for each asset, the left tail dependence parameter is always smaller than the right tail dependence parameter. This represents the probability of the clusters of small volatilities is smaller than the probability of the clusters of large volatilities. In other words, large 
volatilities tend to cluster more often than small volatilities. This result reveals that, in addition to the volatility clustering previously found in the GARCH and SV models, volatility clustering is highly asymmetric, with a higher tendency of clusters of large volatilities than small volatilities. To the best of our knowledge, this result has not been documented in the literature. The calculated t-statistics for the null hypothesis that the left tail dependence parameter is equal to or greater than the right tail dependence against the alternative hypothesis that the left tail dependence parameter is smaller than the right tail dependence is strongly rejected in all cases as indicated by the small p-values. We interpret this result as evidence that the left tail dependence parameter is significantly smaller than the right tail dependence parameter. Therefore, the volatility clustering is significantly asymmetric, i.e., there is a significantly higher tendency for clusters of large volatilities to occur than small volatilities.

The asymmetry in the volatility clustering is consistent with the leverage effect and the return-volatility feedback effect documented in the literature, notably in Bollerslev et al. (2006) for intra-daily volatilities. Based on the leverage effect, a negative return, is more likely to be followed by a higher volatility, than a positive return, then through the volatility feedback effect, the higher volatility is likely to be accompanied by a negative return, which, in turn, through the leverage effect again, is followed by another high volatility.... Thus such a process would suggest clusters of large volatilities. On the other hand, the leverage effect also suggests that a positive return is less likely to be followed by an increase of volatility, suggesting a lower tendency of clusters of small volatilities. 
Interestingly, when comparing the results across different groups of assets, we find that the asymmetry of volatility clusters tend to be the strongest for the large company stocks, followed by the exchange rates and then the stock indices. This might be related to the fact that the stock indices are normally considered to be more stable than the single stocks or exchange rates.

Another interesting question is whether the asymmetric pattern of volatility clusters of the returns series is time varying. To answer this question, we estimate the dynamic tail dependence model specified in equations (7)-(9). The result is presented in Table 3. From this table, we note that the parameters that control the dynamics of the tail dependence are generally statistically significant, providing some evidence of time variation in the degree of volatility clustering in the return series. However we find that in 8 out of 11 cases, the values of the AIC are slightly smaller, while the values of the BIC are larger, in the dynamic models than in the static models. Based on this evidence, we conclude that overall, the static models perform as well as the dynamic models.

To visually assess how the volatility clusters change over time, we plot the dynamics of the volatility tail dependence parameters for the single stocks, exchange rates, and the stock indices in Figure 1, 2, and 3 respectively. In all the figures, the solid line plots the dynamics of the right tail dependence (which is the probability of clusters of large volatilities), the dotted line is the dynamics of the left tail dependence (which is the probability of clusters of small volatilities), while the dashed line is the average of the difference between the right and left tail dependence. First, the right tail dependence line generally lies above the left 
tail dependence line. Thus, over time, the tail dependence is asymmetric, in the sense that large volatility clusters more often than small volatility. This is also visible on the dashed line, which is above the $\mathrm{x}$ axis.

It is well documented that the volatility clustering at the daily or weekly interval is strongly persistent and has a long memory, See Bollerslev et al. (1992) for a survey of this literature for ARCH models and Ray \& Tsay (2000). Thus it would also be interesting to investigate how long the memory lasts and how slowly the volatility clusters die out. To do this, we examine the clusters of volatilities of the return series from the first lag to the $40^{t} h$ lag. We present the results in Table 4 . From the table, we first observe that the degree of volatility clusters decreases gradually as the lag increases. This is reflected in generally smaller tail dependence parameters and larger values of AIC and BIC with the increase of the lag. Second, even after 40 days, the clusters in large volatilities are still statistically significant in most of the cases, indicating a strong persistence in the clusters of large volatilities. Finally, the clusters decay at a slower speed for the exchange rate volatilities than for the stocks and stock indices. Thus volatility clusters are more persistent in foreign exchange markets than stock markets.

To visualize the decaying patterns of the clusters, we plot the left and right tail dependence parameters against the lags for each group in Figure 4, 5 and 6 . These figures confirm that the clusters decay very slowly, showing a strong degree of the clustering persistency. In addition, these figures also show that the decay could alternate in size. For instance, in the case of the GBPA return, the right tail dependence between volatilities of the past 5 days is 
larger than that of the past 4 days.

In summary, we find a strong persistence in the clusters of large volatilities. This is consistent with the long-memory of volatilities documented in the literature. Anderson and Bollerslev (1997) explain the source of the volatility persistence by considering the volatility as a mixture of numerous heterogeneous short-run information arrivals, and the aggregation of these information flow processes may lead to the long memory dependence.

\section{Conclusions}

In this paper, we have analyzed the volatility clustering of a number of return series by using the copula approach with the realized volatility constructed from the high frequency data. The copula approach has made it possible to detect the asymmetry in the clusters of large and small volatilities. The availability of the high frequency data, and, thus, the ability to construct the realized volatility, make it convenient for us to use the copula approach directly.

We found that the volatility clusters in the return series are asymmetric, in the sense that large volatilities tend to cluster more often than small volatilities. This asymmetric volatility clusters were visible even when we allowed for the time variation in the volatility clusters. Finally, we found that the clusters are both strongly persistent and durable in the sense that they do not die out even after a one month period. 


\section{References}

[1] Andersen, T. and Bollerslev T. Heterogeneous information arrivals and return volatility dynamics: uncovering the long-run in high frequency returns. The Journal of Finance 1997, VOL. LII, NO. 3, 975-1006.

[2] Andersen, T.G. and Bollerslev, T. Answering the skeptics: yes, standard volatility models do provide accurate forecasts. International Economic Reviews 1998, Vol. 39, 115-158.

[3] Andersen, T. G., Bollerslev, T. and Diebold, F. X. Roughing it up: Including jump components in the measurement, modeling and forecasting of return volatility. Review of Economics and Statistics 2007, 89, 701-720.

[4] Barndorff-Nielsen, O. E. and Shephard, N. Non-gaussian ornstein-uhlenbeck models and some of their uses in financial economics. The Royal Statistical Sociey B 2001, Vol. 63, $167-241$.

[5] Bollerslev, T., R. Chou and K. F. Kroner. ARCH modeling in finance. Journal of Econometrics 1992, 52, 5-59.

[6] Bollerslev, T., Litvinova, J. and Tauchen, G. Leverage and volatility feedback effects in high-frequency data. Journal of Financial Econometrics 2006, Vo. 4, No. 3, 353-384.

[7] Chen, X. and Fan, Y. Estimation of copula-based semiparametric time series models. Journal of Econometrics 2006, 130, 307-335. 
[8] Cherubini, U., Luciano, E. and Vecchiato, W. Copula Methods in Finance. John Wiley \& Sons, England; 2004.

[9] Chollete, L., de la Peña, V., Lu, C. The scope of international diversification: implications of alternative measures. Working paper, NHH; 2006.

[10] Embrechts, P., McNeil, A. and Strautman, D. Correlation and dependence in risk management: propertites and pitfalls, in Risk Management: Value at Risk and Beyond, ed. M.A.H. Dempster, Cambridge University Press, Cambridge; 2002. 176-223.

[11] Engle, R. F., Bollerslev T. Modelling the persistence of conditional variances. Econometric Reviews 1986, 5, 1-50, X1-87.

[12] Fermanian, J.-D., Scaillet, O. Some statistical pitfalls in copula modeling for finanical appplications, in Capital Formation, Governance and Banking, E. Klein (editor), Nova Science Publishing; 2005.

[13] Genest, C., Ghoudi, K., Rivest, L. A semiparametric estimation procedure of dependence parameters in multivariate families of distributions. Biometrika 1995; 82 (3), 543-552.

[14] Hansen, P. R. and Lunde, A. Realized variance and market microstructure noise. Journal of Business and Economic Statistics 2006, Vol. 12, 371-417.

[15] Hu, L. Dependence patterns across financial markets: a mixed copula approach. Applied Financial Economics 2006, V16, 717-729. 
[16] Joe, H. Multivariate models and dependence concepts. Monographs on statistics and applied probability, 73. London: Chapman and Hall; 1997.

[17] Jondeau, E., Rockinger, M. The copula-GARCH model of conditional dependencies: An international stock market application. Journal of International Money and Finance $2006,25,827-853$.

[18] Koopman, S. J., B. Jungbacker and Hol E. Forecasting daily variablility of the S\&P 100 stock index using historical realized and implied volatility measurements. Journal of Empirical Finance 2005, Vol 12, 445-475.

[19] Maheu, J. M. and McCurdy, T. H. Do high-frequency measures of volatility improve forecasts of return distribution? Forthcoming in Journal of Econometrics 2009.

[20] Martens, M. Measuring and forecasting S\&P 500 index futures volatility using highfrequency data. Journal of Furture Markets 2002, Vol. 22, 497-518.

[21] McAleer, M., Medeiros M.C. Realized volatility: a review. Econometric Reviews 2008, Vol 27, 10-45.

[22] Nelson, R.B. An introduction to copulas, Springer, New York; 1999.

[23] Patton, A.L. Modelling asymmetric exchange rate dependence. International Economic Review 2006, 47(2), 527-556. 
[24] Patton, A.L. Copula-based models for financial time series, Forthcoming Handbook of Financial Time Series, T. G. Andersen, R. A. Davis, J.-P. Kreiss and T. Mikosch (eds), Springer Verlag; 2008.

[25] Ray, B. and Tsay, R. Long-range dependence in daily stock volatilities. Journal of Business and Economic Statistics 2000, 18(2),254-262.

[26] Rodriguez, J. C. Measuring financial contagion: a copula approach. Journal of Empirical Finance 2006, 14, 401-423.

[27] Sklar, A. Fonctions de répartition á n dimensions et leurs marges, Publications de l'Institut de Statistique de l'Université de Paris, 1959, 8, 229-231.

[28] van Dijk, D. and Martens, M. Measuring volatility with the realized range. Journal of Econometrics 2007, 138, 181-207.

[29] Zhang, L. Mykland, P. A., and Ait-Sahalia, Y. A tale of two scales: determining integrated volatility with noisy high frequency data. Journal of the American Statistical Association 2005, vol. 100, 1394-1411. 


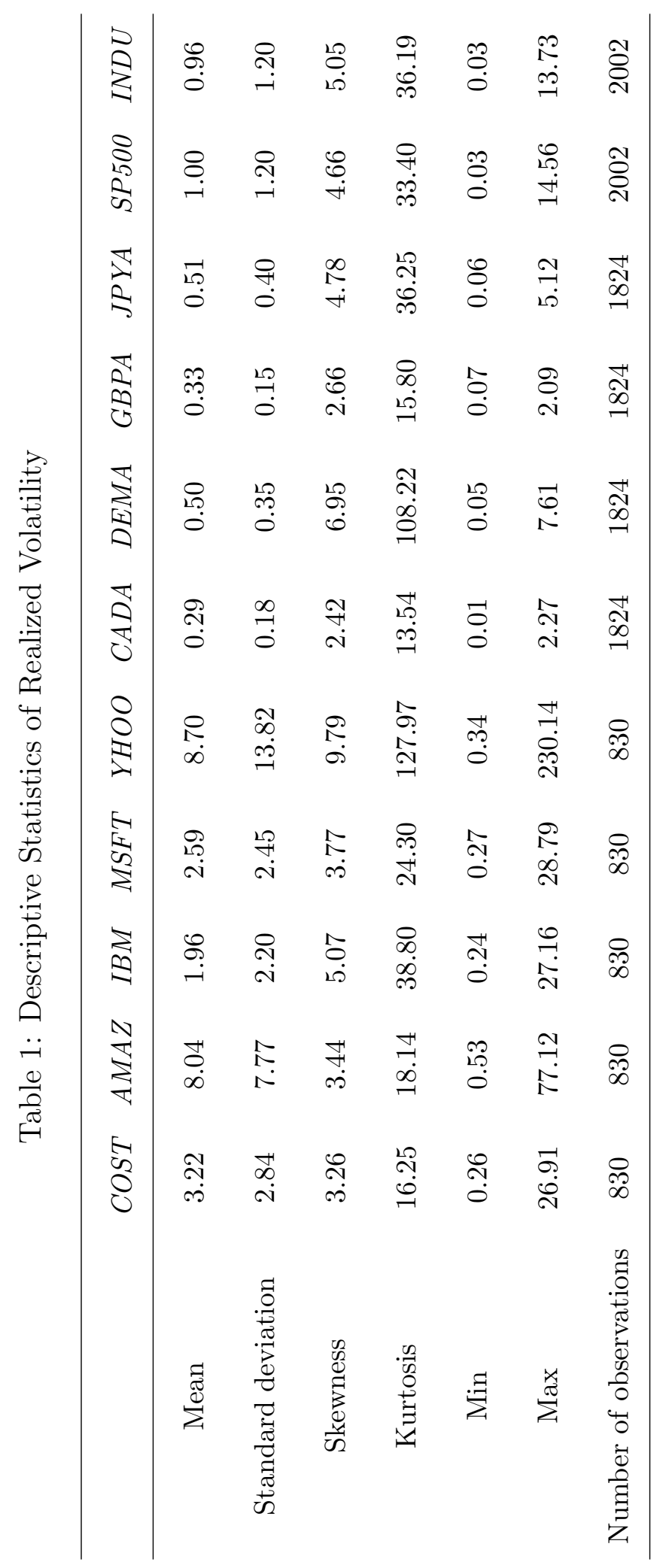




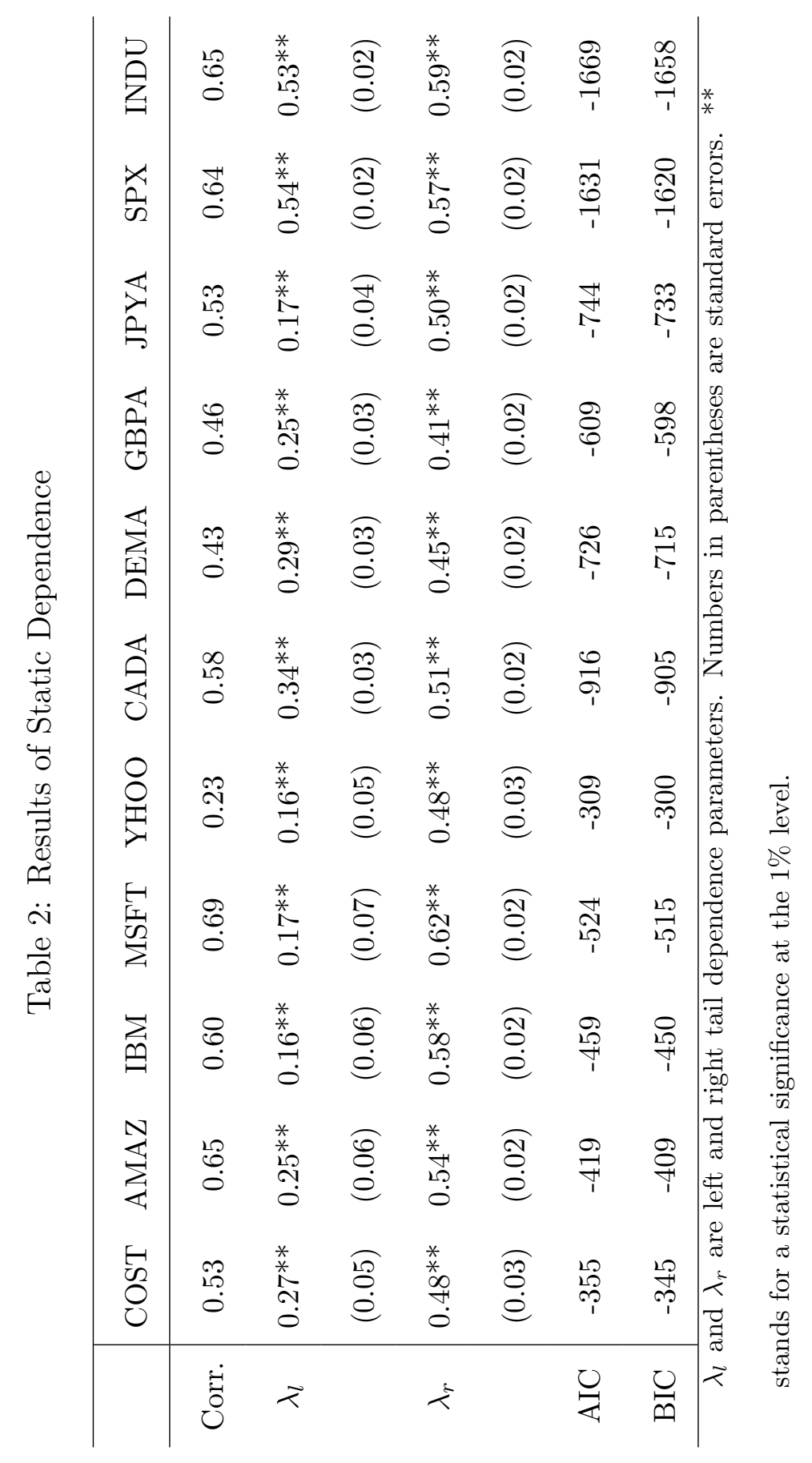




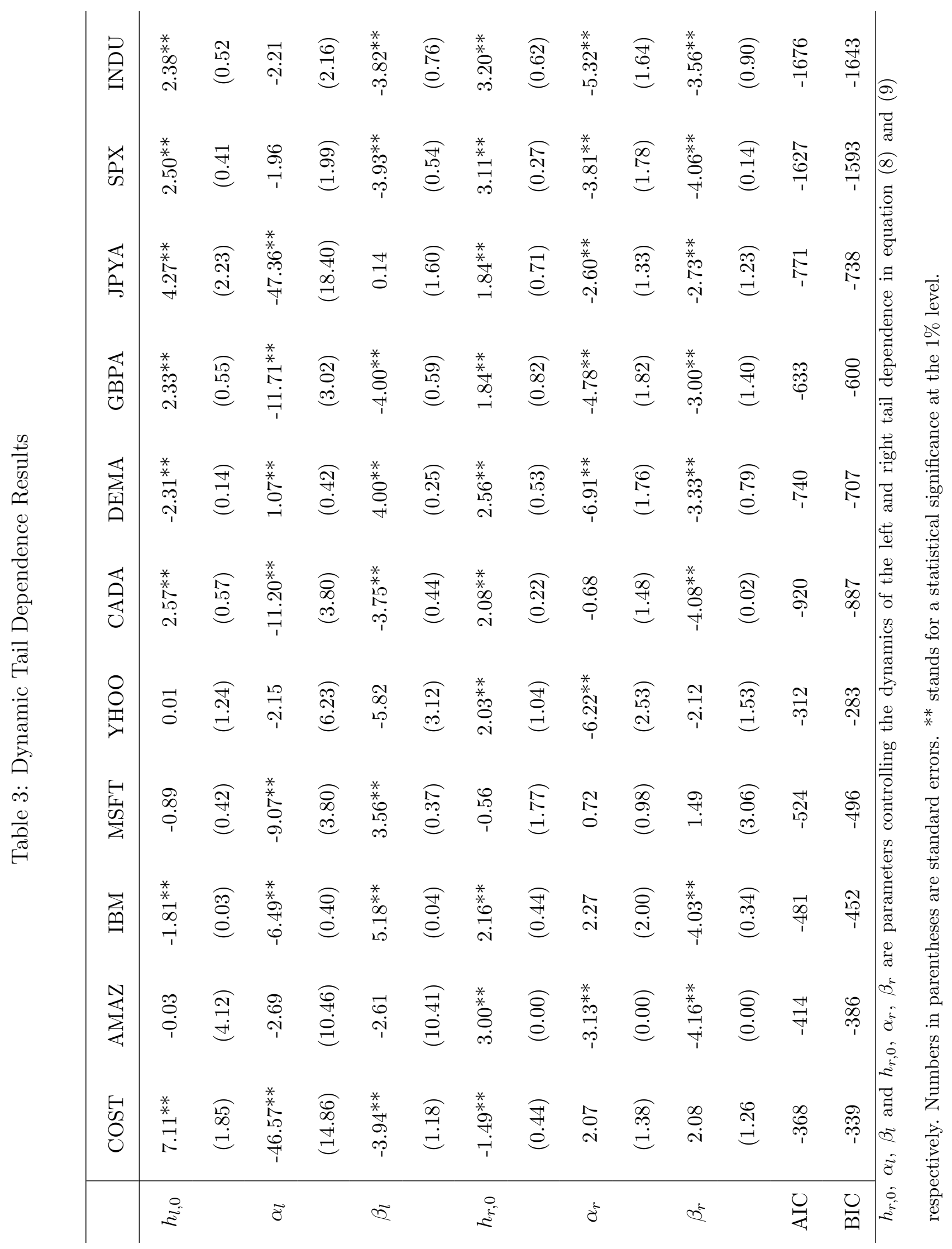




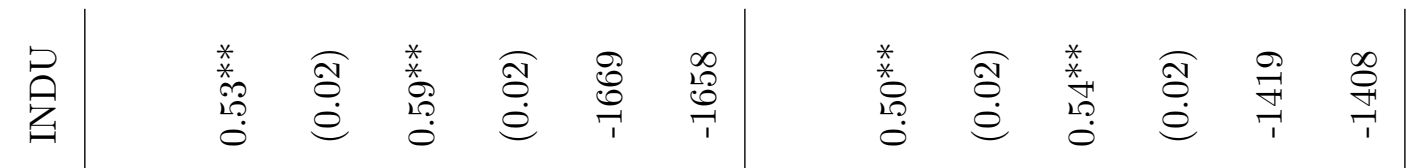

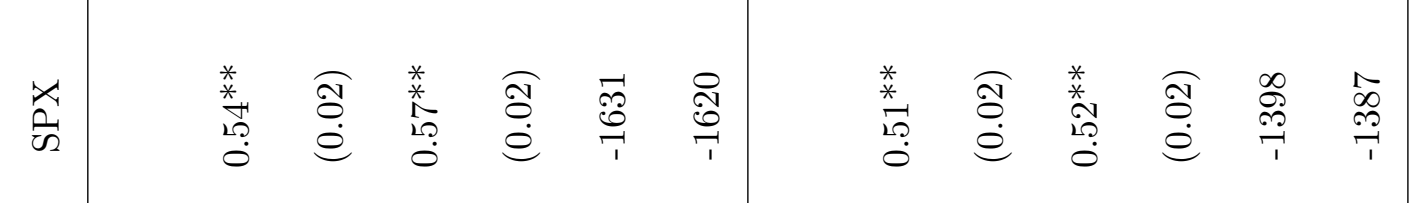

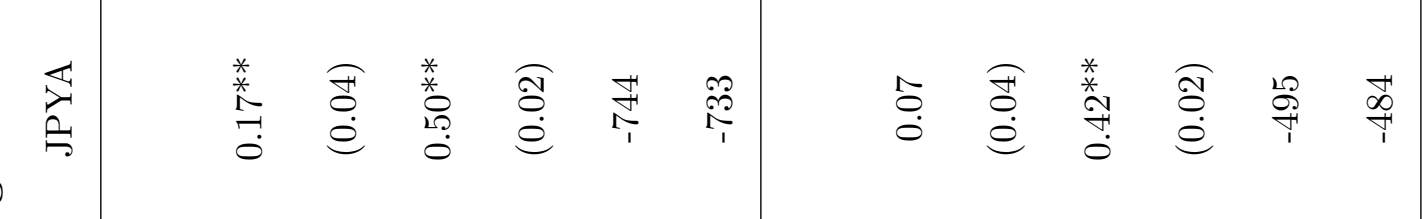

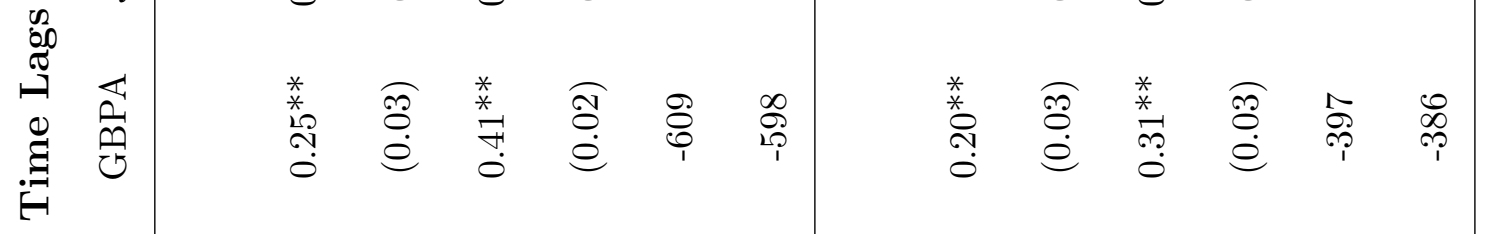

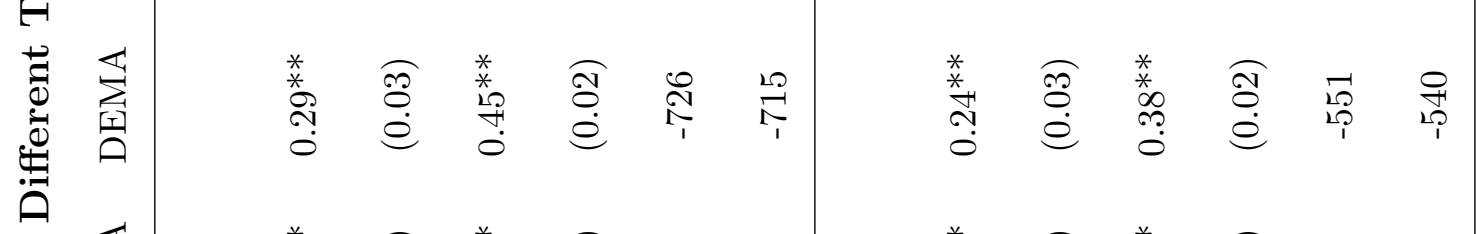

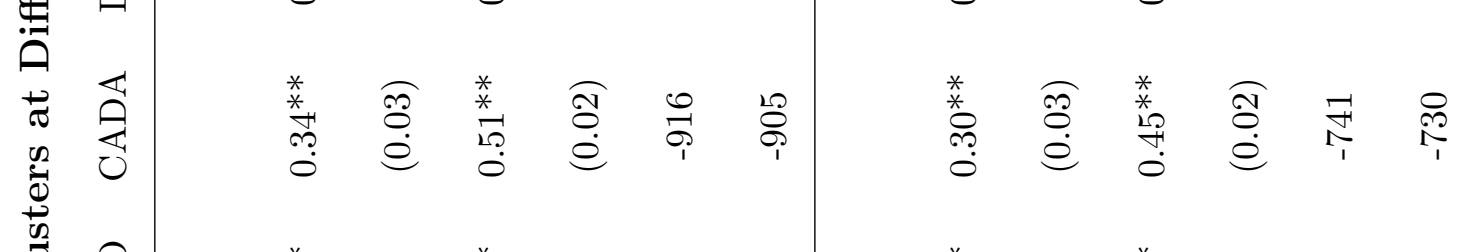

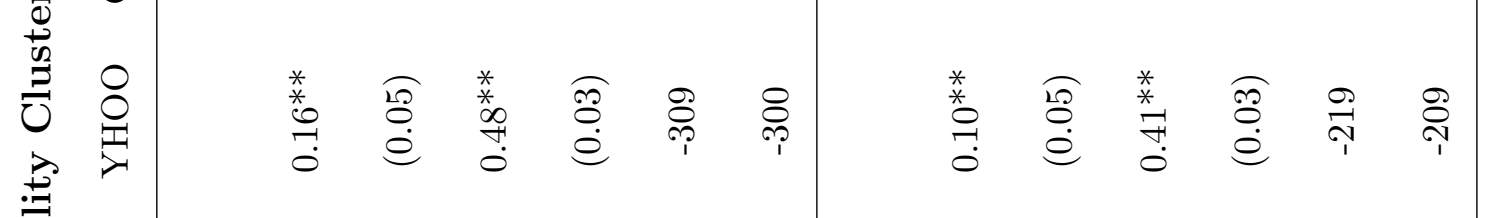

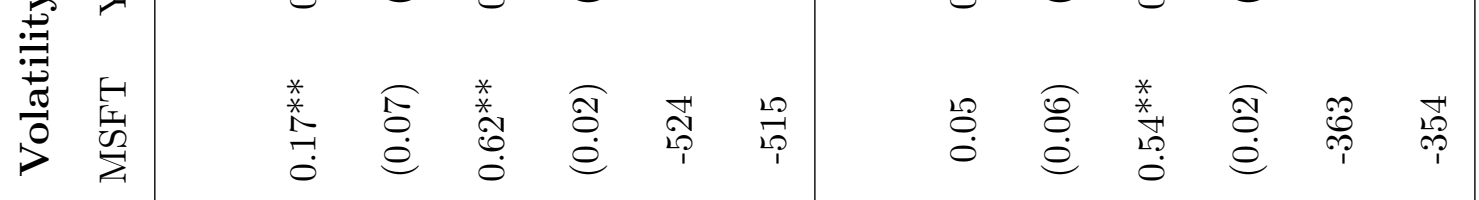

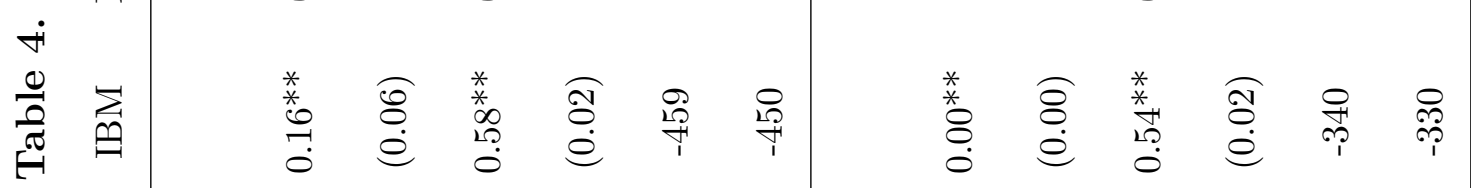

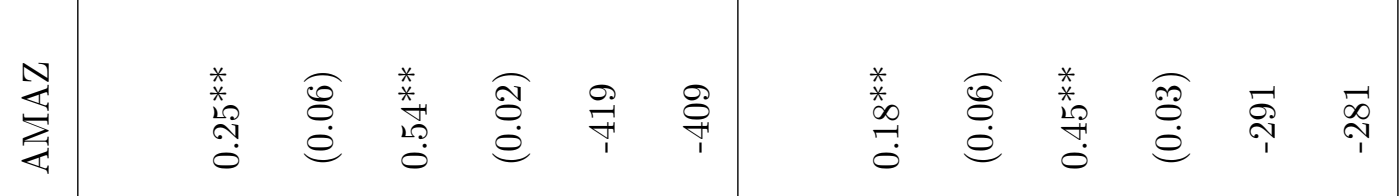

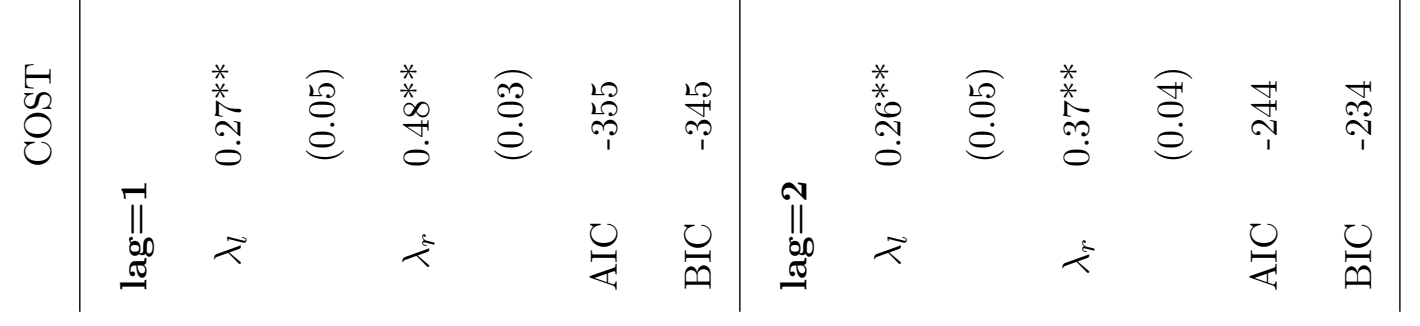




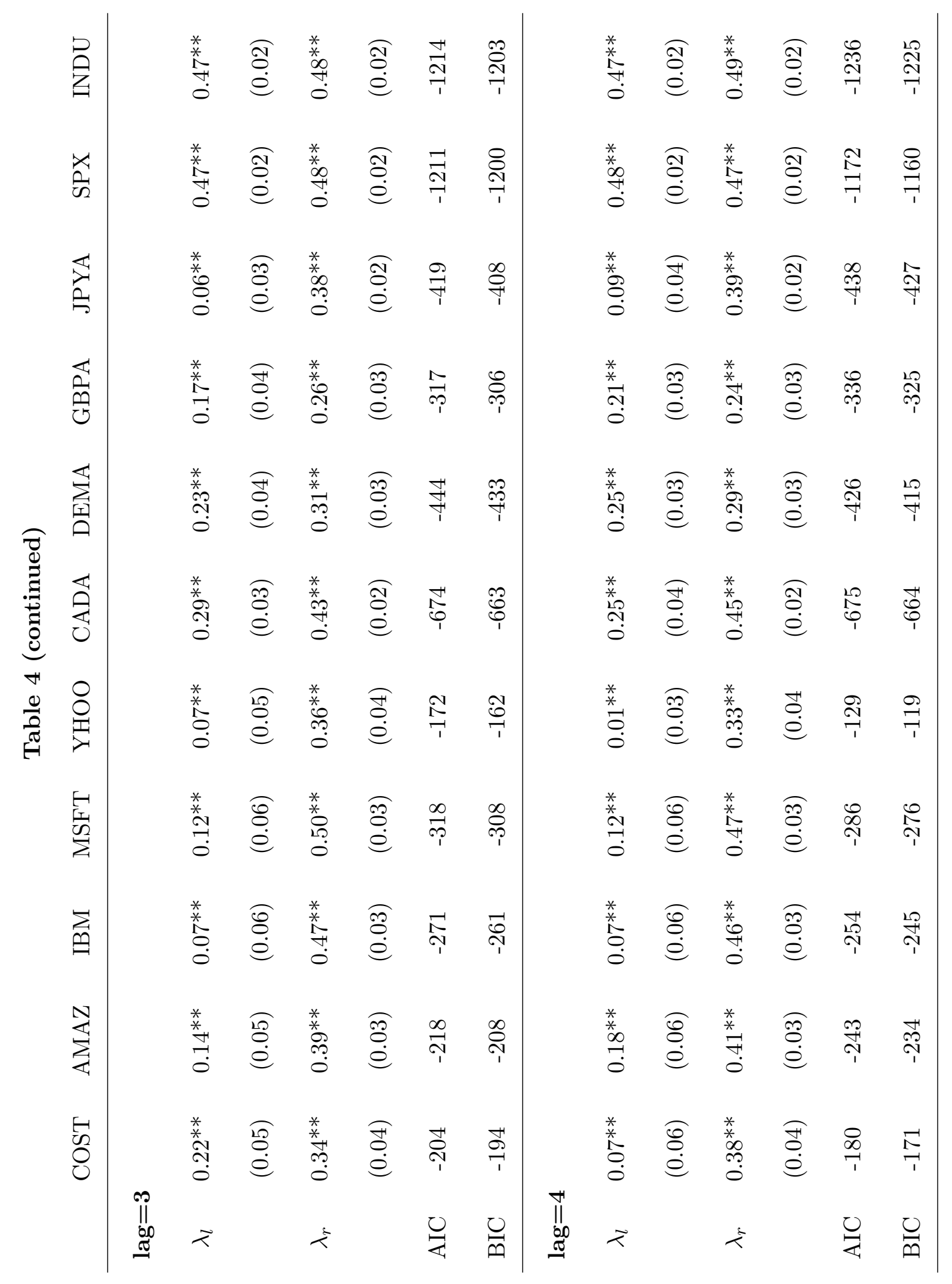




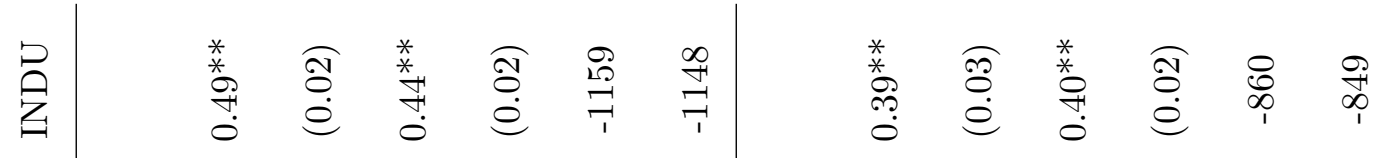

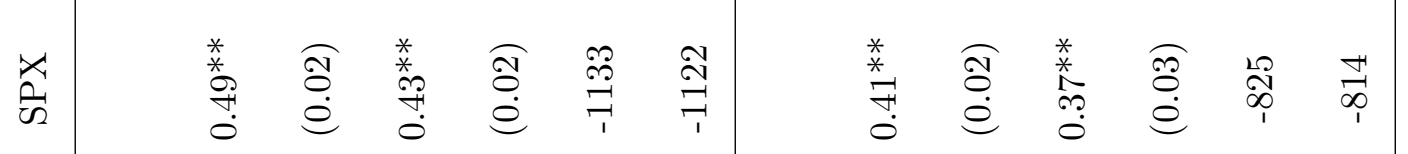

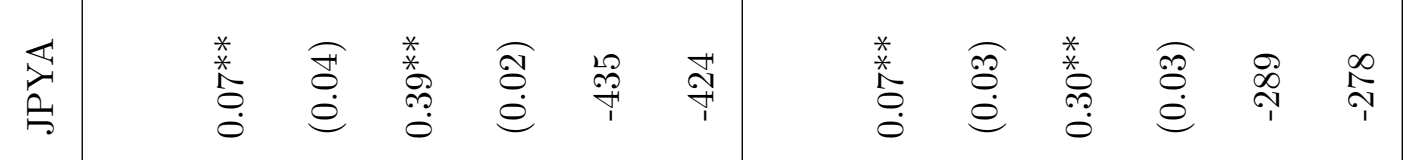

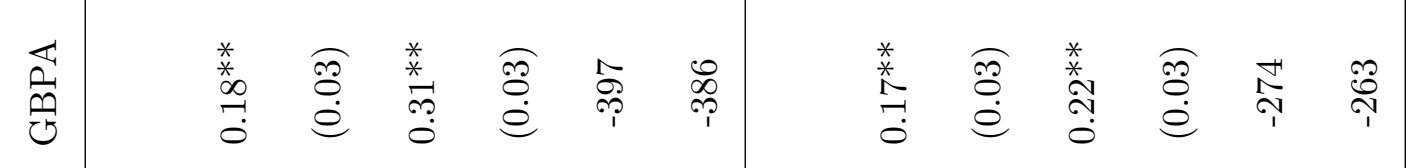

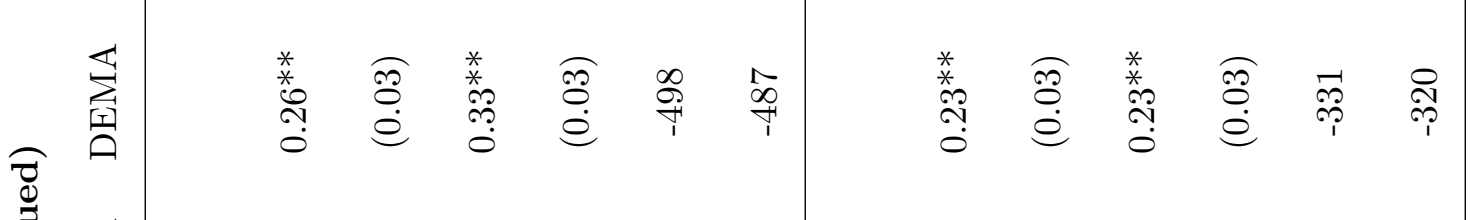

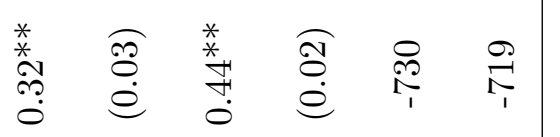

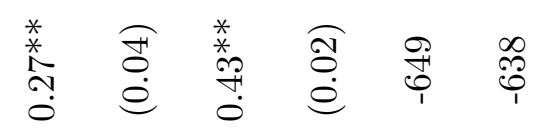

竞递

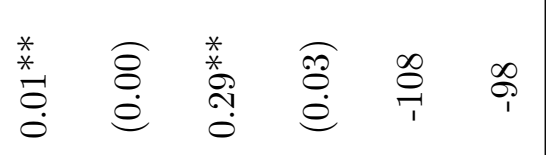

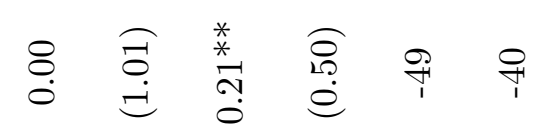

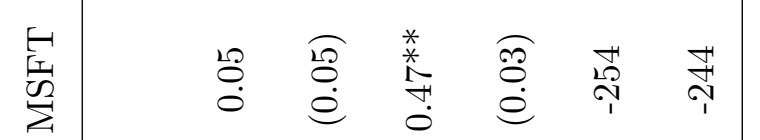

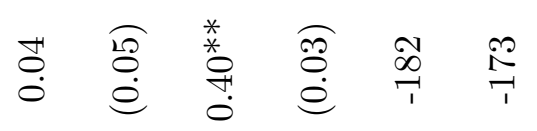

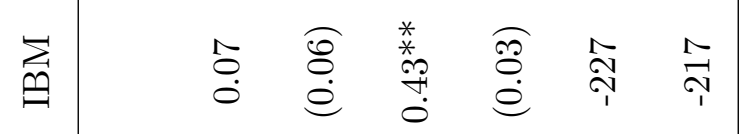

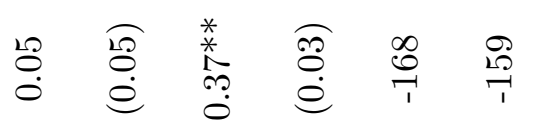

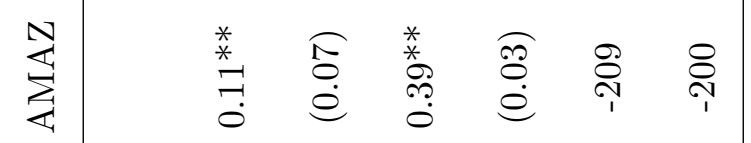

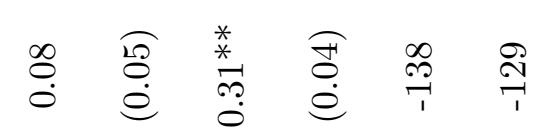

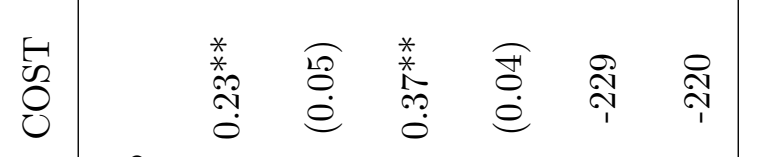

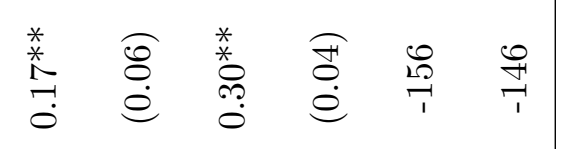

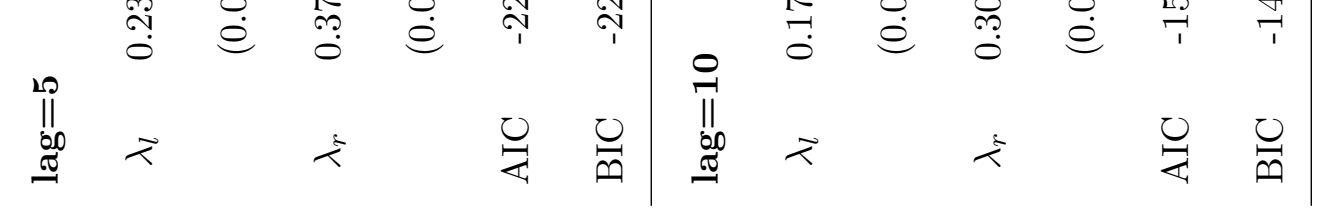




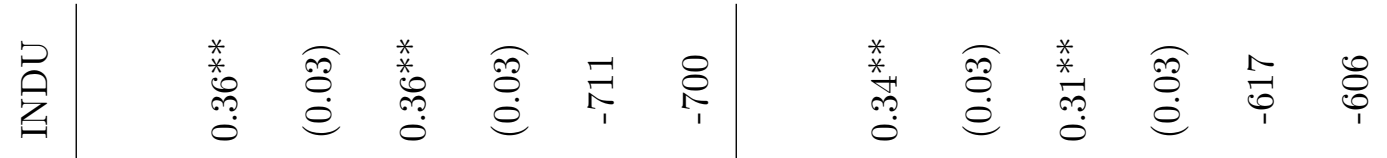

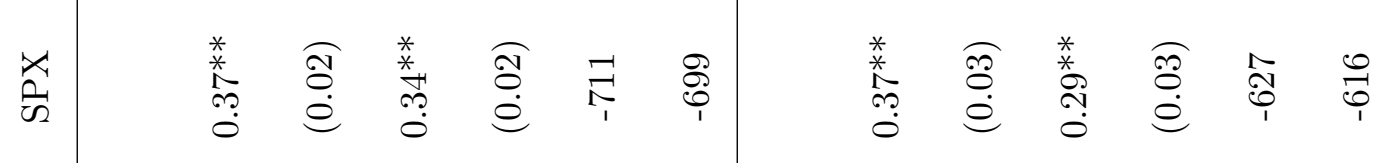

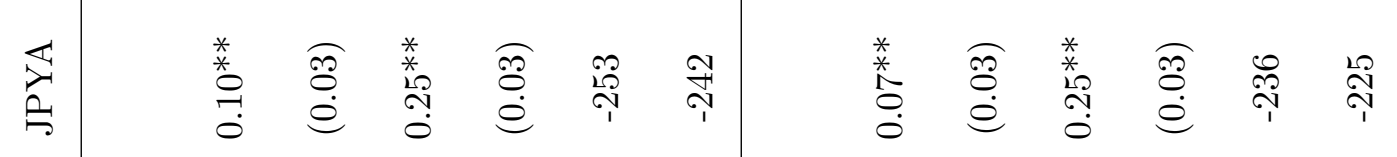

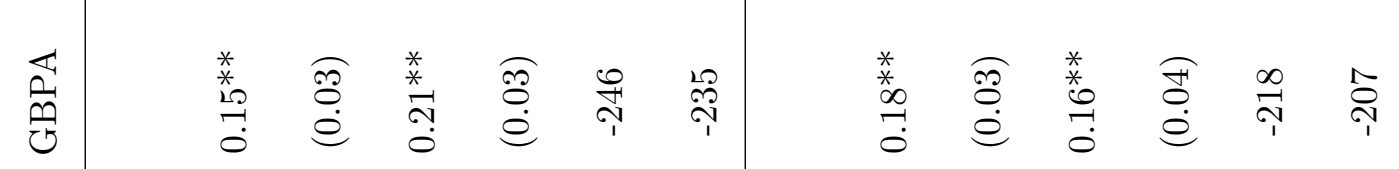

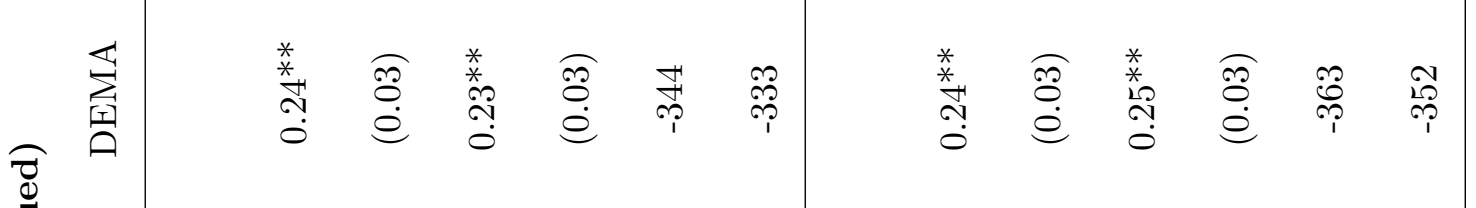

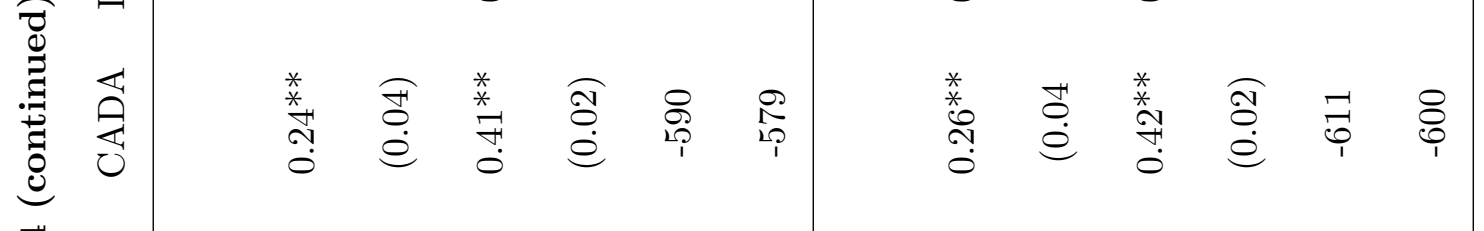

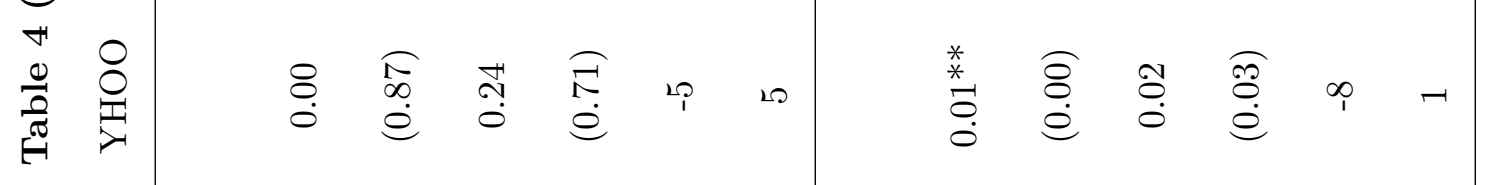

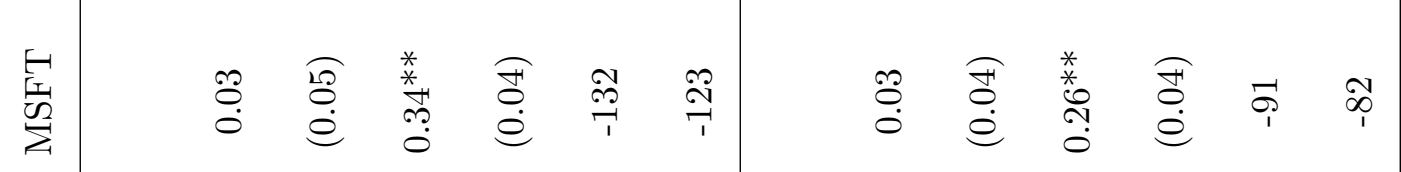

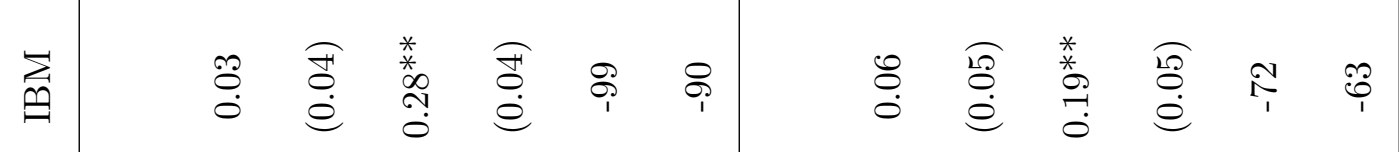

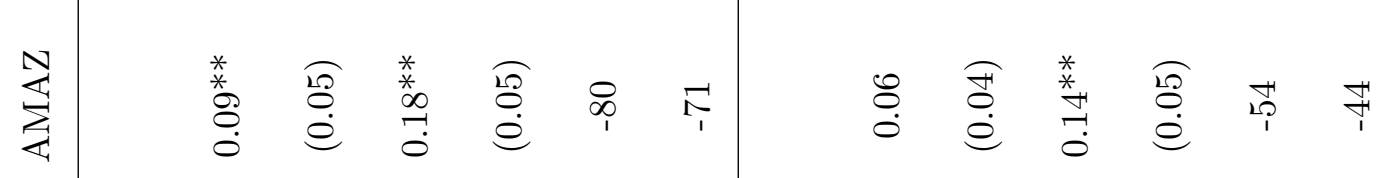

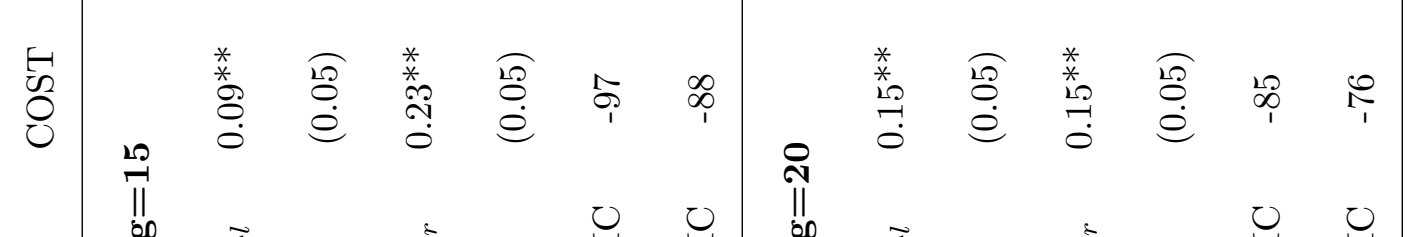

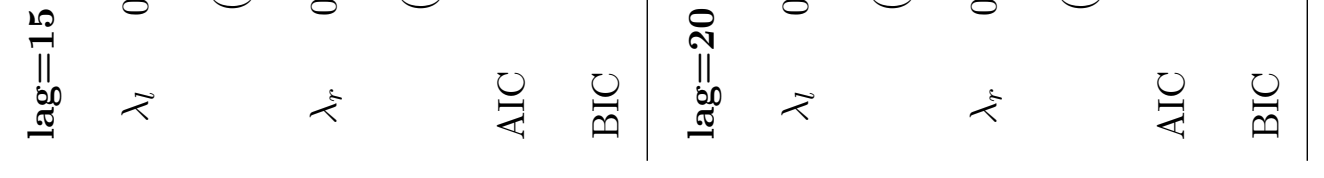




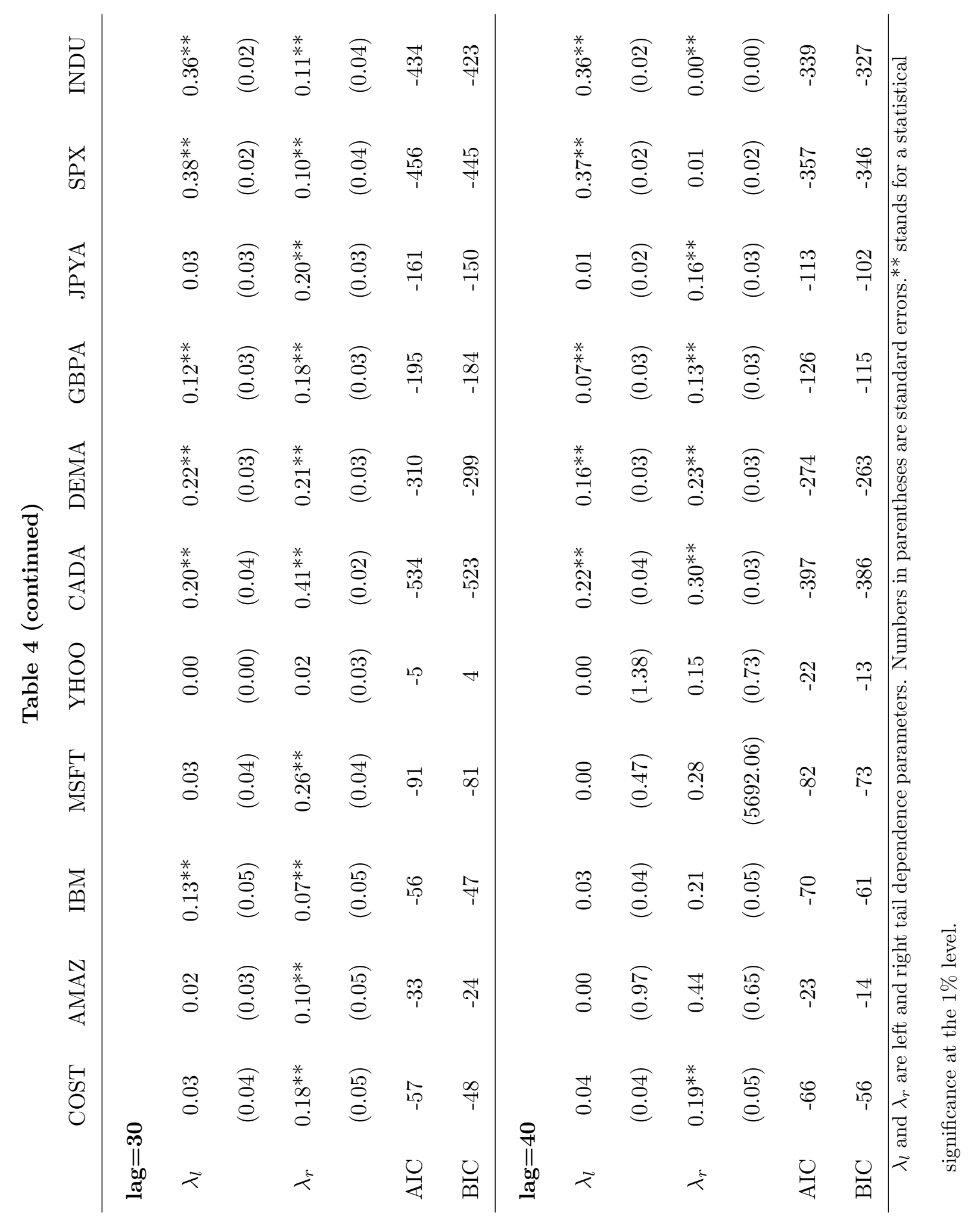


Figure 1: Dynamics of Stock Volatility Clusters
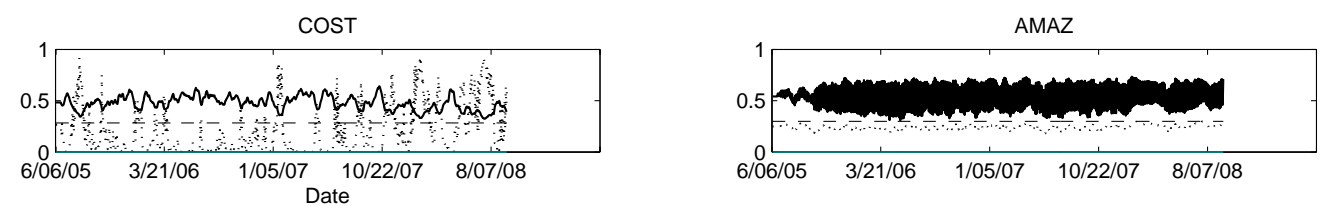

IBM
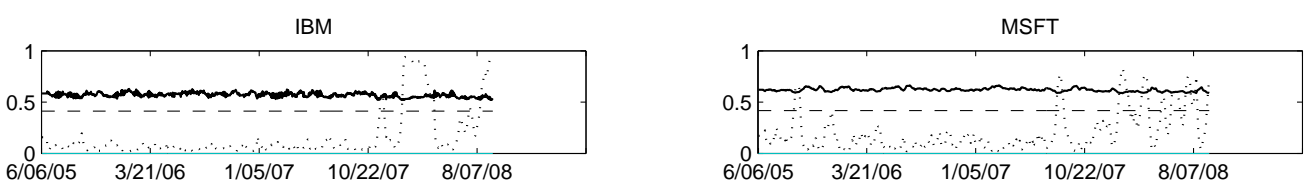

YHOO

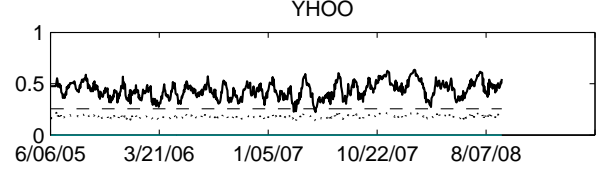

The solid, dotted, and dashed lines plot the dynamics of the right, left tail dependence, and the average of the difference between the right and left tail dependence respectively.

Figure 2: Dynamics of Exchange Rate Volatility Clusters
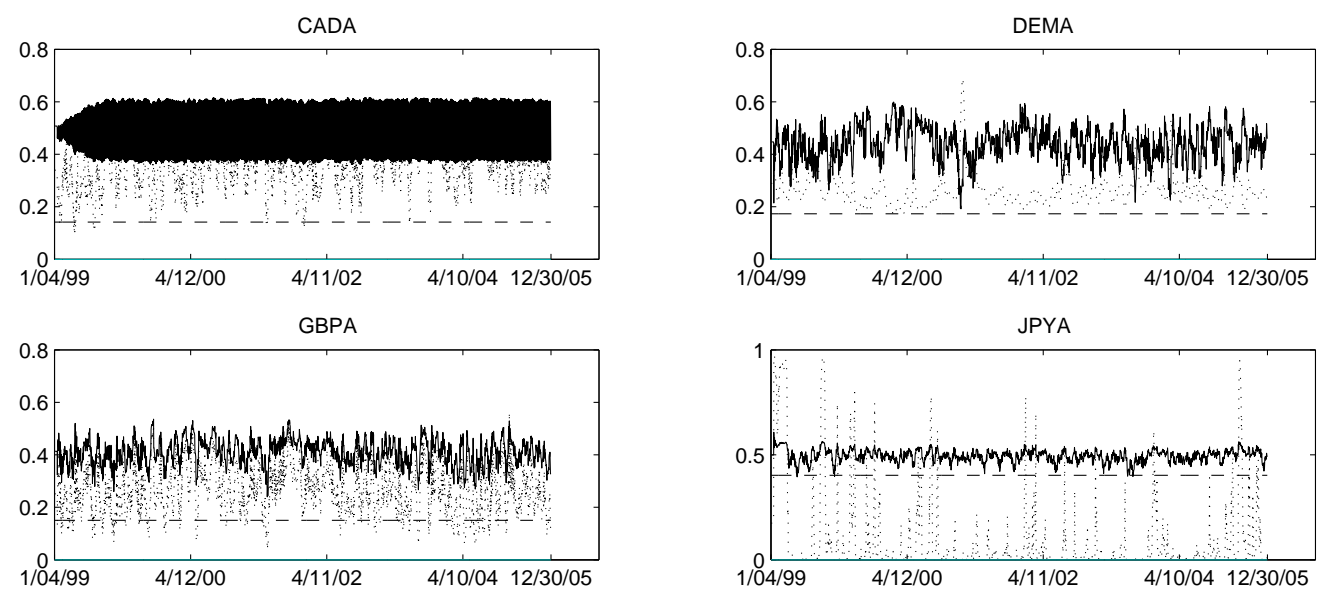

The solid, dotted, and dashed lines plot the dynamics of the right, left tail dependence, and the average of the difference between the right and left tail dependence respectively. 
Figure 3: Dynamics of Stock Indices Volatility Clusters
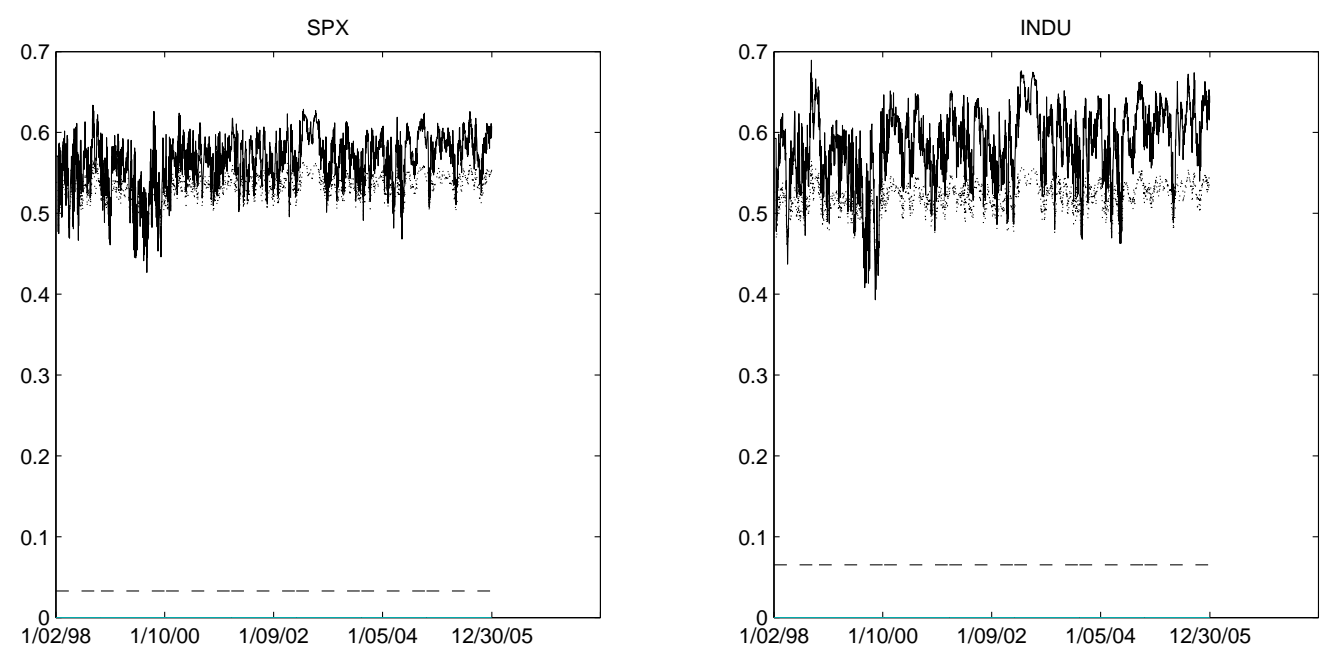

The solid, dotted, and dashed lines plot the dynamics of the right, left tail dependence, and the average of the difference between the right and left tail dependence respectively.

Figure 4: Persistence of Stock Volatility Clusters
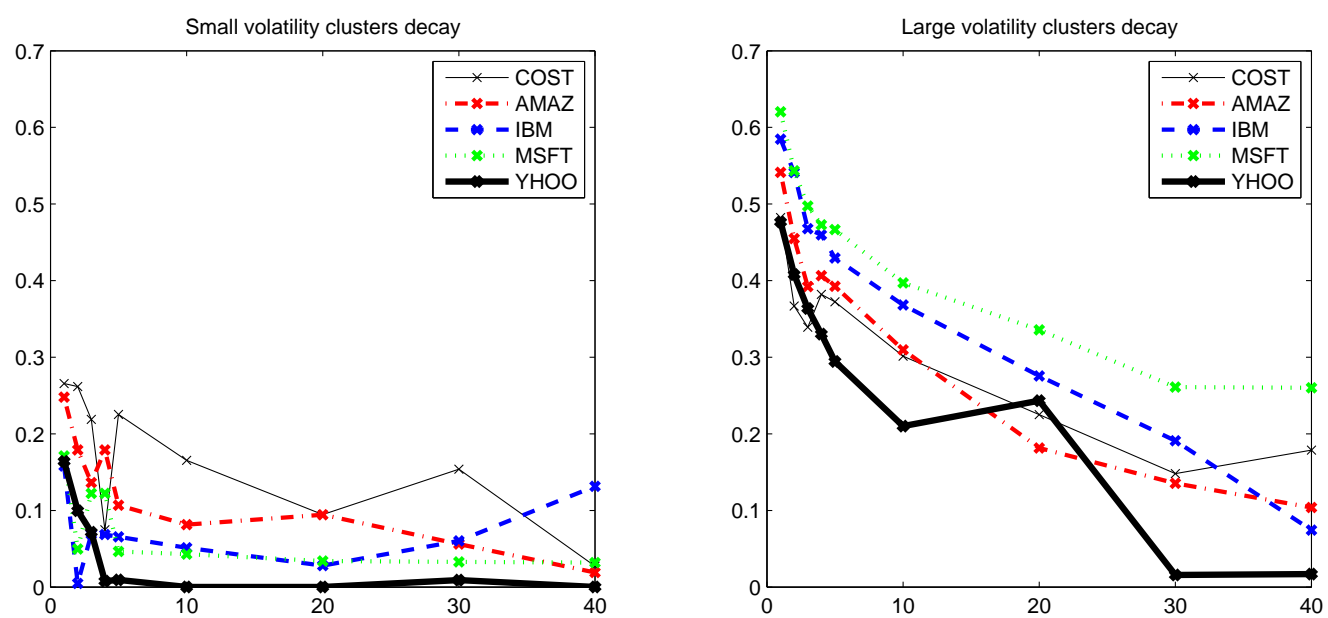

The $\mathrm{x}$ axis marks the day lags, the $\mathrm{y}$-axis is for the volatility tail dependence between day 1 and day $\mathrm{T}$ marked on $\mathrm{x}$ axis. 
Figure 5: Persistence of Exchange Rate Volatility clusters
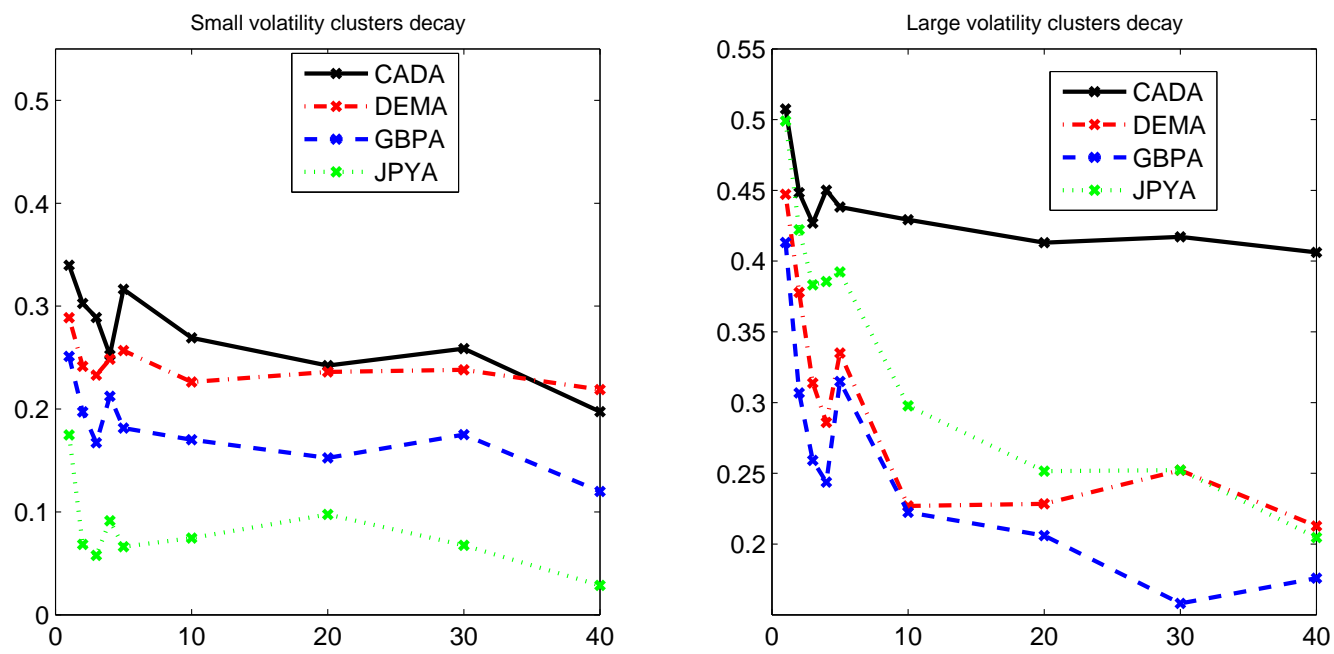

The $\mathrm{x}$ axis marks the day lags, the $\mathrm{y}$-axis is for the volatility tail dependence between day 1 and day $\mathrm{T}$ marked on $\mathrm{x}$ axis.

Figure 6: Persistence of Stock Indices Volatility clusters
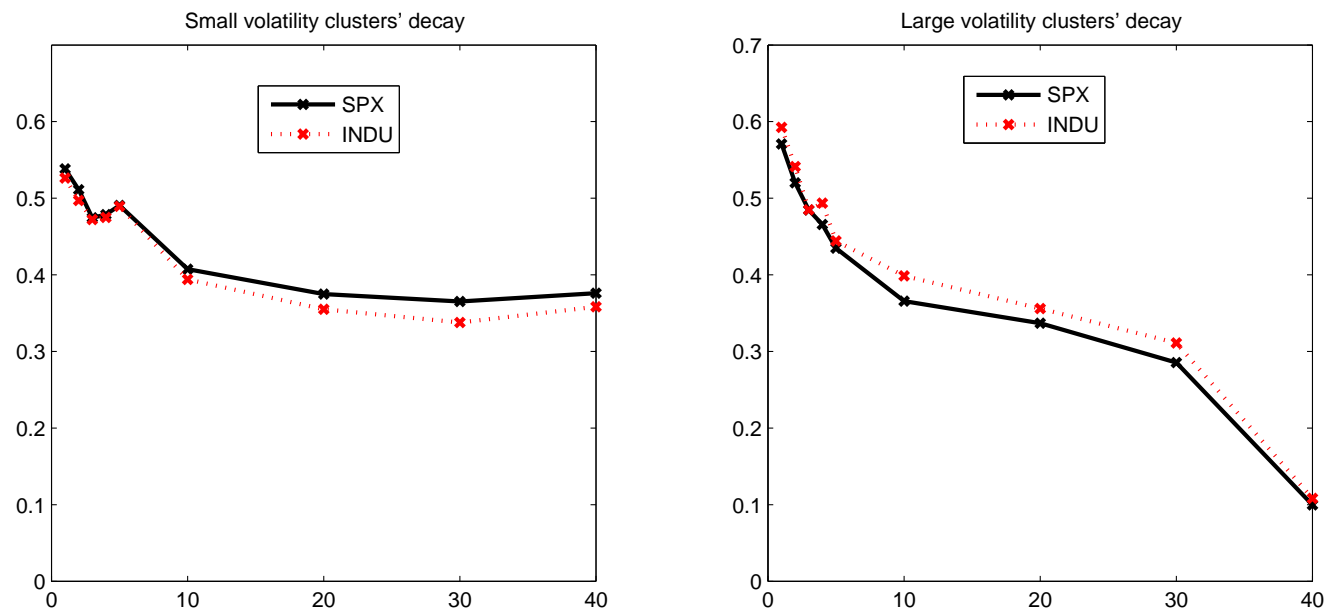

The $\mathrm{x}$ axis marks the day lags, the $\mathrm{y}$-axis is for the volatility tail dependence between day 1 and day $\mathrm{T}$ marked on $\mathrm{x}$ axis. 